\title{
Quality factor of an electrically small magnetic dipole antenna with magneto-dielectric
} core

Kim, Oleksiy S.; Breinbjerg, Olav

Published in:

2010 URSI International Symposium on Electromagnetic Theory

Link to article, DOI:

10.1109/URSI-EMTS.2010.5637320

Publication date:

2010

Document Version

Publisher's PDF, also known as Version of record

Link back to DTU Orbit

Citation (APA):

Kim, O. S., \& Breinbjerg, O. (2010). Quality factor of an electrically small magnetic dipole antenna with magnetodielectric core. In 2010 URSI International Symposium on Electromagnetic Theory IEEE.

https://doi.org/10.1109/URSI-EMTS.2010.5637320

\section{General rights}

Copyright and moral rights for the publications made accessible in the public portal are retained by the authors and/or other copyright owners and it is a condition of accessing publications that users recognise and abide by the legal requirements associated with these rights.

- Users may download and print one copy of any publication from the public portal for the purpose of private study or research.

- You may not further distribute the material or use it for any profit-making activity or commercial gain

- You may freely distribute the URL identifying the publication in the public portal 


\title{
Quality Factor of an Electrically Small Magnetic Dipole Antenna with Magneto-Dielectric Core
}

\author{
Oleksiy S. Kim ${ }^{1}$ and Olav Breinbjerg ${ }^{2}$ \\ Department of Electrical Engineering, Technical University of Denmark \\ $\emptyset$ rsteds Plads, Building 348, DK-2800, Kgs.Lyngby, Denmark \\ 1 oskeelektro.dtu.dk \\ 2 obdelektro.dtu.dk
}

\begin{abstract}
In this work, we investigate the radiation $Q$ of electrically small magnetic dipole antennas with magneto-dielectric core versus the antenna electrical size, permittivity and permeability of the core. The investigation is based on the exact theory for a spherical magnetic dipole antenna with material core.
\end{abstract}

\section{INTRODUCTION}

An exact theory of an ideal spherical magnetic dipole antenna with magneto-dielectric core was presented in [1], [2]. The theory proves that a magnetic core introduced into an impressed spherical electric current radiating the $\mathrm{TE}_{10}$ spherical mode diminishes the internal stored magnetic energy, and thus reduces the antenna $Q$. Furthermore, for a finite-size antenna there is an optimum permeability of the core $\mu^{\text {opt }}$ that ensures the lowest quality factor $Q_{\min }=Q\left(\mu^{\mathrm{opt}}\right)$, and the value of this optimum permeability is not infinite as it is the case for an infinitely small antenna. In [1], it was also shown that the $Q_{\min }$ increases with the dielectric permittivity $\varepsilon$ of the core. Consequently, the $Q_{\min }$ for $\varepsilon$ equal to the free-space permittivity represents the lower bound for the quality factor of a magnetic dipole antenna with magneto-dielectric core [3].

In this work, we perform a detailed investigation of the behavior of the $Q$ of a spherical magnetic dipole antenna versus the antenna size as well as the permittivity $\varepsilon$ and permeability $\mu$ of the core.

\section{THEORY}

Consider an impressed electric surface current density $\mathbf{J}$ on a surface of a spherical material core of radius $a$. It can be shown that to radiate the elementary magnetic dipole field (the $\mathrm{TE}_{10}$ spherical mode) the current must be of the form

$$
\mathbf{J}=\hat{\mathbf{r}}_{\phi} J_{0} \sin \theta
$$

where $\hat{r}_{\phi}$ is the azimuthal unit vector. In this case, its radiation quality factor $Q$ can be expressed as

$$
Q=\max \left\{Q_{\mathrm{H}}, Q_{\mathrm{E}}\right\}
$$

where $Q_{\mathrm{H}}$ and $Q_{\mathrm{E}}$ are the quality factors due to the stored magnetic and electric energies, respectively. Using the exact expressions in [1] these are written as

$$
Q_{\mathrm{H}}=\left\{1+\frac{1}{\mu_{\mathrm{r}}}\left(\frac{1-\left(k_{s} a\right) j_{0}\left(k_{s} a\right) j_{-1}\left(k_{s} a\right)}{2 j_{1}^{2}\left(k_{s} a\right)}-1\right)\right\} Q_{\mathrm{Chu}}
$$

$$
\begin{aligned}
& Q_{\mathrm{E}}=\left\{\frac{(k a)^{2}}{(k a)^{2}+1}\right. \\
& \left.+\frac{1}{\mu_{\mathrm{r}}}\left(\frac{1+\left(k_{s} a\right) j_{0}\left(k_{s} a\right) j_{-1}\left(k_{s} a\right)-2 j_{0}^{2}\left(k_{s} a\right)}{2 j_{1}^{2}\left(k_{s} a\right)}\right)\right\} Q_{\mathrm{Chu}} \text { (4) }
\end{aligned}
$$

where $k_{s} a=\sqrt{\mu_{\mathrm{r}} \varepsilon_{\mathrm{r}}} k a, k$ is the free-space wave number, $\mu_{\mathrm{r}} \in$ $\Re$ and $\varepsilon_{\mathrm{r}} \in \Re$ are the relative permeability and permittivity of the core, respectively, $j_{n}(v)$ is the spherical Bessel function of order $n$, and $Q_{\text {Chu }}$ is the Chu lower bound

$$
Q_{\mathrm{Chu}}=\frac{1}{(k a)^{3}}+\frac{1}{k a} \text {. }
$$

In the range, where the stored magnetic energy exceeds the stored electric energy [3], we can point out two important particular cases.

1) Vanishing electrical size $k a$. It is easy to see that as $k a \rightarrow 0$ expression (3) reduces to the well-known approximation by Wheeler [4]

$$
Q=Q_{\mathrm{H}} \approx\left(1+\frac{2}{\mu_{r}}\right) Q_{\mathrm{Chu}}, k a \ll 1 .
$$

2) Pure dielectric core. For a non-magnetic core $\left(\mu_{\mathrm{r}}=1\right)$, expression (3) simplifies as

$Q=Q_{\mathrm{H}}=\left\{\frac{1-\left(k_{d} a\right) j_{0}\left(k_{d} a\right) j_{-1}\left(k_{d} a\right)}{2 j_{1}^{2}\left(k_{d} a\right)}\right\} Q_{\mathrm{Chu}}, \mu_{\mathrm{r}}=1$

where $k_{d} a=\sqrt{\varepsilon_{\mathrm{r}}} k a$.

\section{PROPERTIES OF Q VS. $\mu_{\mathrm{R}}, \varepsilon_{\mathrm{R}}$, AND $k a$}

Generally, the radiation $Q$ (2) is a function of three independent variables - the antenna electrical size $k a$, the relative permittivity $\varepsilon_{\mathrm{r}}$ and the permeability $\mu_{\mathrm{r}}$ of the core. It requires a four-dimensional space to graphically represent the function $Q\left(k a, \varepsilon_{\mathrm{r}}, \mu_{\mathrm{r}}\right)$. On the other hand, the ratio $Q / Q_{\text {Chu }}$ can be conveniently described by only two independent variables $k_{d} a$ and $\mu_{\mathrm{r}}$ and therefore directly visualized in the $\left(k_{d} a, \mu_{\mathrm{r}}\right)$ space, as shown in Fig. 1.

The ratio $Q / Q_{\mathrm{Chu}}$ appears as a sequence of valleys separated by singularities of resonances in the magneto-dielectric core. As follows from expressions (3)-(4), the location of the singularities is described by a simple equation

$$
k_{s} a=v_{n}
$$




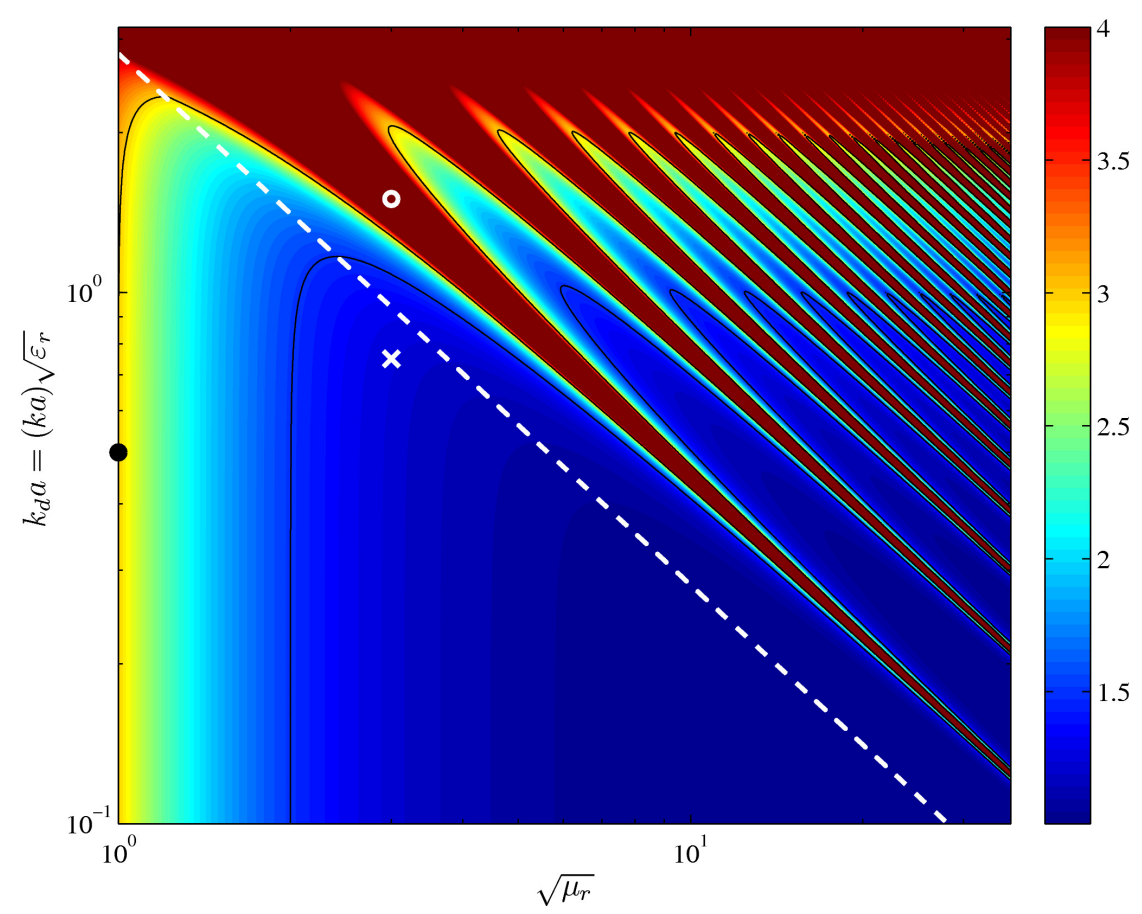

Fig. 1. The ratio $Q / Q_{\text {Chu }}$ for a magnetic dipole antenna with magneto-dielectric core. The white dashed line shows the optimal parameters of the core according to (9).

where $v_{n}$ are zeroes of the spherical Bessel function $j_{1}(v)$, $n=1,2,3, \ldots$

In all the valleys, the $Q$ reaches the Chu lower bound as $k_{d} a \rightarrow 0$ subject to $\mu_{\mathrm{r}} \rightarrow \infty$. However, for a given $k_{d} a$, the lowest $Q$ is achieved in the first, most extensive valley [3]. It is demonstrated numerically in [1] and proved rigorously in [3], that the permeability $\mu_{\mathrm{r}}$ satisfying

$$
\mu_{\mathrm{r}}=\frac{u_{1}^{2}}{\left(k_{d} a\right)^{2}}=\frac{u_{1}^{2}}{\varepsilon_{\mathrm{r}}(k a)^{2}}
$$

ensures the lowest possible $Q$ provided the antenna size $k a$ and permittivity $\varepsilon_{\mathrm{r}}$ of the core are fixed. The constant $u_{1} \approx 2.816$ in (9) is the first solution of equation (7) in [3].

Since the ratio $Q / Q_{\text {Chu }}$ tends to decrease with $\varepsilon_{\mathrm{r}}$ away from the resonances, it is advantageous to select the permittivity of the core as low as possible. In the limiting case $\varepsilon_{\mathrm{r}}=1$, by substituting (9) into (3), we obtain the lower bound for the radiation $Q$ of electrically small magnetic dipole antennas with magneto-dielectric core [3]. In Fig. 1, this bound is represented by the values $Q / Q_{\mathrm{Chu}}$ along the white dashed line corresponding to the expression (9).

\section{PRACTICAL Aspects}

In practice, pure magnetic materials are hardly available. Moreover, it is difficult to acquire a material that satisfies the optimal parameters in (9) for a given antenna size. Such a material would require custom design and fabrication. When the range of available materials is limited, in some cases it might be preferable to abandon the core at all. For instance, assume we have a material with parameters $\varepsilon_{\mathrm{r}}=9.0$ and $\mu_{\mathrm{r}}=9.0$. If the desired antenna electrical size is $k a=0.25$, then the ratio $Q / Q_{\mathrm{Chu}}=1.25$, which is close to the optimum, as marked with a white cross in Fig. 1. However, if $k a=0.5$, we hit the resonance, and, consequently, the ratio $Q / Q_{\mathrm{Chu}}=$ $2.6 \cdot 10^{4}$ is extremely high (white circle in Fig. 1). Obviously, in this case the air core is a better choice, since it yields the ratio $Q / Q_{\text {Chu }}=3.0$ (black circle in Fig. 1).

\section{CONCLUSION}

We have discussed properties of the radiation $Q$ of magnetic dipole antennas with magneto-dielectric core using the exact theory derived in [1], [3]. The understanding of the dependencies of $Q$ on the antenna size as well as on the core material parameters is essential for the design of optimal electrically small magnetic dipole antennas.

\section{ACKNOWLEDGMENT}

This work is supported by the Danish Research Council for Technology and Production Sciences within the TopAnt project (http://www.topant.dtu.dk).

\section{REFERENCES}

[1] O. S. Kim, O. Breinbjerg, and A. D. Yaghjian, "Electrically small magnetic dipole antennas with quality factors approaching the Chu lower bound," IEEE Trans. Antennas Propagat., to be published.

[2] O. Breinbjerg and O. S. Kim, "Minimum Q electrically small spherical magnetic dipole antenna - theory," in Proc. Int. Symp. Ant. Propagat. (ISAP 2009), Bangkok, Thailand, October 20-29 2009.

[3] O. S. Kim and O. Breinbjerg, "Lower bound for the radiation Q of electrically small magnetic dipole antennas," submitted to IEEE Trans. Antennas Propagat.

[4] H. A. Wheeler, "The spherical coil as an inductor, shield, or antenna," Proc. IRE, vol. 46, no. 9, pp. 1595-1602, 1958. 